\title{
Analytical Microscopy Techniques in Support of Nanoparticle Environmental Safety \& Health Considerations
}

\author{
K. L. Bunker, T. L. Lersch, and G. S. Casuccio \\ R J Lee Group, Inc., Monroeville, PA 15146
}

Nanotechnology and the use of engineered nanoparticles is a rapidly developing area of materials science. Nanotechnology is a platform technology that enables the creation of new products and processes across a wide variety of industry sectors. Research and development are underway to develop lighter and stronger materials, new battery and fuel cell materials, optical sensors, environmental remediation, food packaging, drug delivery systems as well as a host of other applications. While research is ongoing, nanoscale materials are already being used and sold commercially in electronics, cosmetics, clothing, appliances, and automotive and medical products. It is estimated that there are more than 1,000 manufacturer-identified nanotechnology-based consumer products currently on the market [1].

As research continues and consumer products are already in use, there are concerns related to potential unforeseen health or environmental effects. There is very little exposure data to engineered nanomaterials reported in the scientific literature and therefore the development of nanotechnology safety and health guidelines has been slow. A significant amount of research and development will be needed to quantify the risks to humans, animals, and the ecosystem from the release of nanoparticles. However, we have built a foundation in research methods, characterization techniques, analytical instrumentation, and control strategies. Electron microscopy is one tool that can be used to effectively observe, characterize and better understand nanoparticles and nanomaterials. The health experts are evaluating particle number, size, size distribution, mass, surface area, and composition as possible exposure metrics. Electron microscopy can provide valuable information related to these metrics and has been suggested as a useful technique in the evaluation of nanomaterials in the area of environmental health and safety [2-3].

The work presented will include examples of how electron microscopy imaging and elemental mapping can be used in the characterization of nanomaterials (Fig. 1). In addition, several case studies will be presented, including one from Lawrence Berkeley National Laboratory (LBNL). LBNL has initiated a study to evaluate worker exposure and potential environmental release of unbound engineered nanoparticles (UNP) related to various research activities being performed in their laboratory. The study is being performed to help identify and manage potential health and safety hazards as well as environmental impacts related to UNP. A key component of the study is the characterization of source materials to assist in the determination of worker exposure and environmental release (Fig. 2). This presentation will provide an overview of the LBNL study with a focus on the methodologies being used to analyze the samples [4].

\section{References:}

[1] Woodrow Wilson International Center of Scholars (www.nanotechproject.org/inventories/consumer).

[2] Nanoscience and Nanotechnologies: Opportunities and Uncertainties, The Royal Society and The Royal Academy of Engineering, London, 2004.

[3] National Nanotechnology Initiative: Strategy for Nanotechnology-Related Environmental, Health, and Safety Research, February 2008. 
[4] G. Casuccio et al., Worker and Environmental Assessment of Potential Unbound Engineered Nanoparticle Releases: Phase I and Phase II Final Reports, RJLG and LBNL, September 2009.
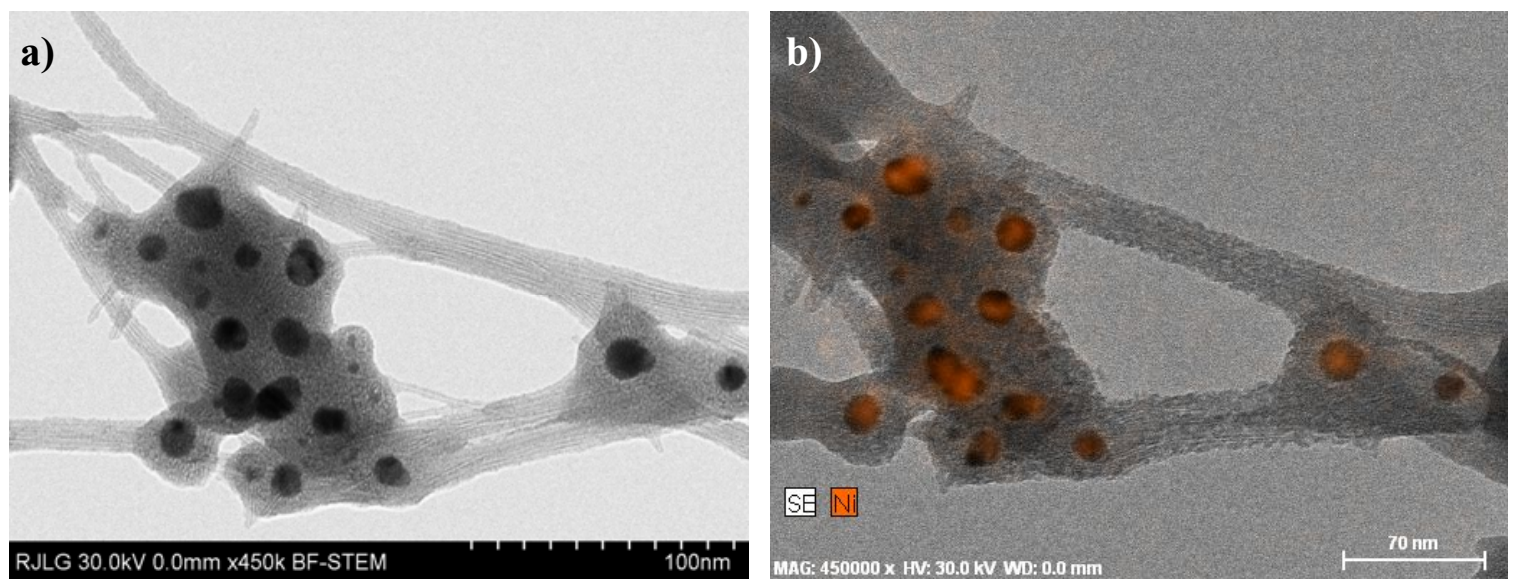

Fig. 1: Electron microscopy images of single-wall carbon nanotubes, including (a) a bright fieldSTEM image of the internal structure and (b) an elemental map showing the composition of the catalyst particles to be nickel.

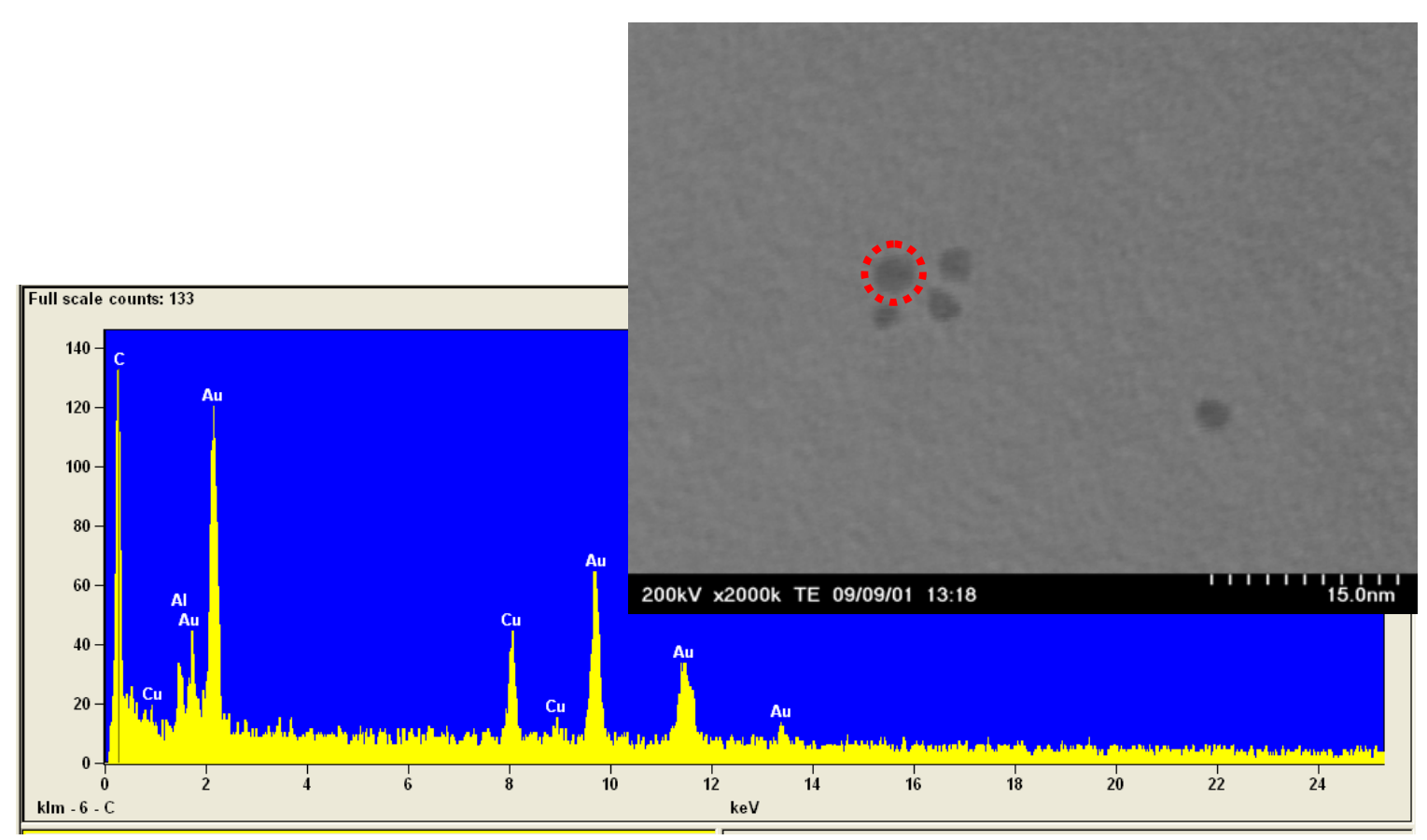

Fig. 2: A bright field-STEM image of $5 \mathrm{~nm}$ gold nanoparticles and a corresponding energy dispersive X-ray spectroscopy spectrum showing the gold peaks. 